\title{
Effect of pairing correlations on incompressibility and symmetry energy in nuclear matter and finite nuclei
}

\author{
E. Khan, ${ }^{1}$ J. Margueron, ${ }^{1}$ G. Colò, ${ }^{2}$ K. Hagino, ${ }^{3}$ and H. Sagawa ${ }^{4}$ \\ ${ }^{1}$ Institut de Physique Nucléaire, Université Paris-Sud, IN2P3-Centre National de la Recherche Scientifique, F-91406 Orsay CEDEX, France \\ ${ }^{2}$ Dipartimento di Fisica, Università degli Studi and Istituto Nazionale di Fisica Nucleare Sezione di Milano, \\ Via Celoria 16, I-20133 Milano, Italy \\ ${ }^{3}$ Department of Physics, Tohoku University, 980-8578 Sendai, Japan \\ ${ }^{4}$ Center for Mathematics and Physics, University of Aizu, Aizu-Wakamatsu, 965-8580 Fukushima, Japan
}

(Received 10 May 2010; published 31 August 2010)

\begin{abstract}
The role of superfluidity in incompressibility and in symmetry energy is studied in nuclear matter and finite nuclei. Several pairing interactions are used: surface, mixed, and isovector dependent. Pairing has a small effect on the nuclear matter incompressibility at saturation density, but the effects are significant at lower densities. The pairing effect on the centroid energy of the isoscalar giant monopole resonance (GMR) is also evaluated for $\mathrm{Pb}$ and $\mathrm{Sn}$ isotopes by using a microscopic constrained-HFB approach and is found to change at most by $10 \%$ the nucleus incompressibility $K_{A}$. It is shown by using the local density approximation that most of the pairing effect on the GMR centroid comes from the low-density nuclear surface.
\end{abstract}

DOI: 10.1103/PhysRevC.82.024322

PACS number(s): 21.10.Re, 21.65.-f, 21.60.Jz

\section{INTRODUCTION}

The nuclear incompressibility and the symmetry energy are closely related to the isoscalar giant monopole resonance (GMR) [1,2] and to the isovector giant dipole resonance (GDR) [3], respectively. The question of the effect of pairing correlations on the centroid energy of the GMR was first addressed in Ref. [4] and has recently known a renewed interest $[5,6]$. In both the $\mathrm{Sn}$ and $\mathrm{Pb}$ isotopic chains, a specific increase of the GMR energy, associated with the corresponding finite nucleus incompressibility $K_{A}$, has been predicted for the doubly magic ${ }^{132} \mathrm{Sn}$ and ${ }^{208} \mathrm{~Pb}$ nuclei [6,7]. A part of this apparent stiffness of doubly magic nuclei may be related to pairing effects that in fact decrease the GMR energy in open-shell nuclei. However, this study has been undertaken only with a pure surface pairing interaction. It is therefore relevant to analyze more systematically this effect using various pairing functionals. It should be noted that the surface versus mixed nature of the pairing interaction is still under discussion. For instance, a recent systematic study based on the odd-even mass staggering seems to slightly favor a surface type of pairing interaction [8].

The apparent decrease of incompressibility in superfluid nuclei raises a question about a possible similar effect in infinite nuclear matter: Until now, the nuclear matter incompressibility has been evaluated by neglecting the pairing part of the functional. However, considering results for finite nuclei, the equations of state used for neutron stars and supernovae predictions should take into account pairing effects in the calculation of the incompressibility modulus. Therefore the question of the behavior of $K_{\infty}$ with respect to the pairing gap is raised because it seems clear from nuclear data that the finite nucleus incompressibility $K_{A}$ decreases with an increasing pairing gap [6]. A similar study for nuclear matter as well as a more systematic study in finite nuclei should be undertaken. This is the goal of the present work. It should also be noted that we will not consider the neutron-proton
$T=0$ pairing channel because the nuclei considered are far from $N=Z$.

The density dependence of the symmetry energy is one of the most debated issues in nuclear physics at present. In fact, this has relevant implications (1) for nuclear structure, because it has an important effect on the size of the neutron rms radius in neutron-rich nuclei; (2) for nuclear reactions, for example, in intermediate-energy heavy-ion collisions where the isospin distribution of the reaction products is dictated by the density dependence of the symmetry energy; and obviously, (3) for the description of neutron stars. Review papers have been devoted to this topic $[9,10]$. Empirical information on the symmetry energy can be obtained from various sources, none of which so far is conclusive by itself. No measurement of the neutron skin is available that is accurate enough to constrain the symmetry energy. The properties of the isovector GDR, of the low-lying electric dipole excitations, and of the charge-exchange spin-dipole strength have been suggested as constraints (see, e.g., Ref. [11]). In addition, different model analyses of heavy-ion collisions have been proposed as a test of the main trend of the symmetry energy at densities below saturation. However, in none of these studies, to our knowledge, has the problem of the pairing effects on the symmetry energy been addressed.

In this article, the effects of the pairing correlations on incompressibility and on symmetry energy are studied consistently in nuclear matter and in finite nuclei. The effects coming from the correlation energy associated with the pairing force are included. These pairing effects are studied in Sec. II. In Sec. III, a local density approximation (LDA) approach to the problem is employed to understand the connection between the effects in infinite matter and finite systems: The ${ }^{120} \mathrm{Sn}$ nucleus is used as a benchmark. Finally, in Sec. IV, a microscopic study of the role of superfluidity in the incompressibility of finite nuclei is undertaken, employing several pairing interactions: surface, mixed, and isovector dependent. Section V concludes. 


\section{NUCLEAR MATTER}

In this section, we study the effects of the pairing correlations on the incompressibility and the symmetry energy in nuclear matter.

\section{A. Energy density}

The nuclear energy density $\epsilon(\epsilon / \rho=E / V)$ is the sum of the Skyrme part, $\epsilon_{\text {Skyrme }}$, that includes the kinetic energy [12], plus the pairing energy density:

$$
\epsilon=\epsilon_{\text {Skyrme }}+\epsilon_{\text {pair }} \text {. }
$$

Here

$$
\epsilon_{\text {pair }}=-\frac{1}{2}\left(N_{n} \Delta_{n}^{2}+N_{p} \Delta_{p}^{2}\right) .
$$

In Eq. (2), $\Delta_{\tau}$ is the pairing gap and $N_{\tau}$ is the density of states, given by $N_{\tau}=m_{\tau}^{*} k_{F \tau} / \pi^{2} \hbar^{2}$, with $\tau=n, p$. The energy density $\epsilon$ is a function of the total density $\rho$ and of the asymmetry parameter $\delta=\left(\rho_{n}-\rho_{p}\right) / \rho$. In the $T=1$ channel, several pairing interactions are defined by

$$
v_{\text {pair }}^{\text {IS }}\left(\vec{r}, \vec{r}^{\prime}\right)=v_{0}\left[1-\eta\left(\frac{\rho}{\rho_{0}}\right)^{\alpha}\right] \delta\left(\vec{r}-\vec{r}^{\prime}\right),
$$

as a function of the value of $\eta$ that can range from 0 (volume-type pairing) to 1 (surface-type pairing). In Eq. (3), the parameter $\alpha$ is set to 1 , and $\rho_{0}$ is taken as the saturation density of symmetric nuclear matter throughout all the study; moreover, we adopt the parameters $\eta=0.35$ and 0.65 for the volume-surface mixed-type pairing interactions and $\eta=1.0$ for the surface-type interaction. The values of $v_{0}$ in all these cases are adjusted, for each $\eta$, in such a way as to obtain equivalent results for the two-neutron separation energy in the Sn isotopes by HFB calculations with the SLy5 parameter set. The pairing cutoff energy is set at $60 \mathrm{MeV}$ [13]. These values of $v_{0}$ are given in Table I. In the following, these pairing interactions will be denoted as IS because they depend on the isoscalar density.

We have also considered pairing interactions having the isovector density dependence with $\delta=\left(\rho_{n}-\rho_{p}\right) / \rho$ in addition to the isoscalar density dependence. The MSH interaction is defined as [16]

$$
\begin{aligned}
v_{\text {pair }}^{\mathrm{MSH}}\left(\vec{r}, \vec{r}^{\prime}\right)= & v_{0}\left[1-(1-\delta) \eta_{s}\left(\frac{\rho}{\rho_{0}}\right)^{\alpha_{s}}-\delta \eta_{n}\left(\frac{\rho}{\rho_{0}}\right)^{\alpha_{n}}\right] \\
& \times \delta\left(\vec{r}-\vec{r}^{\prime}\right)
\end{aligned}
$$

TABLE I. Strength $v_{0}$ (in $\mathrm{MeV} \cdot \mathrm{fm}^{3}$ ) of the pairing interactions obtained in the case of various Skyrme functionals. The values of $v_{0}$ are adjusted, for each $\eta$, to obtain equivalent results to those of Ref. [13] for the two-neutron separation energy in the $\mathrm{Sn}$ isotopes by the HFB calculations with the parameter set SLy5. The energy cutoff for the pairing window is taken to be $60 \mathrm{MeV}$.

\begin{tabular}{lccc}
\hline \hline & $\eta=0.35$ & $\eta=0.65$ & $\eta=1.00$ \\
\hline SLy5 [12] & -285 & -390 & -670 \\
Sk255 [14] & -265 & -390 & -600 \\
Sk272 [14] & -265 & -390 & -600 \\
LNS [15] & -250 & -390 & -670 \\
\hline \hline
\end{tabular}

with $v_{0}=-448 \mathrm{MeV} \cdot \mathrm{fm}^{3}, \eta_{s}=0.598, \alpha_{s}=0.551, \eta_{n}=$ 0.947 , and $\alpha_{n}=0.554$ (with a cutoff energy of $60 \mathrm{MeV}$ ). The YS interaction also has the isospin dependence as [17]

$$
\begin{aligned}
v_{\text {pair }}^{\mathrm{YS}}\left(\vec{r}, \vec{r}^{\prime}\right)= & v_{0}\left[1-\left(\eta_{0}+\eta_{1} \tau_{3} \delta\right) \frac{\rho}{\rho_{0}}-\eta_{2}\left(\delta \frac{\rho}{\rho_{0}}\right)^{2}\right] \\
& \times \delta\left(\vec{r}-\vec{r}^{\prime}\right),
\end{aligned}
$$

with $v_{0}=-344 \mathrm{MeV} \cdot \mathrm{fm}^{3}, \eta_{0}=0.5, \eta_{1}=0.2$, and $\eta_{2}=2.5$ (with a cutoff energy of $50 \mathrm{MeV}$ ). The parameters mentioned have been used in connection with the SLy5 interaction. In the following, these pairing interactions will be denoted as IS + IV.

Effective interactions in the pairing channel are faced to the double counting problem [18]. However, using a zero-range interaction constrained on bare interactions allows us to avoid this problem [19]. This motivates the use of a different interaction in the pairing channel compared to the particle-hole one. The pairing interactions used in this work are of two types: either fitted on BCS gaps in symmetric and neutron matter calculated with bare interaction, as in the MSH case, or designed to fit observables in nuclei, as in the IS and YS cases. These pairing interactions are featured with zero range and with a cutoff, following the prescription of Ref. [19]: Our aim is to provide reasonable pairing descriptions in nuclei to use the same interaction in nuclei and in nuclear matter. The MSH interaction is considered as an extension of Ref. [19], including isospin dependence. It has been shown that it is possible to study the surface properties of the pairing interaction using slabs of nuclear matter [20,21]. However, it should be mentioned that one usually deals with only the first order in the diagrammatic expansion of the many-body equations. Thus adjusting the pairing gap on the bare interaction is done at this level and is therefore perfectible: There are screening effects that are at next order, for instance. Therefore adjusting the gaps on the bare interaction is complementary to other ways such as constraining the pairing interaction to fit the gaps in finite nuclei. We use both approaches, as explained earlier.

In this article, symmetric nuclear matter is studied, as is the behavior of the symmetry energy. With this purpose, it should be noted that the MSH interaction is obtained by constraining the neutron and proton gaps of the bare interaction in both symmetric and pure neutron matter. The YS interaction is adjusted on several nuclei with different isospins, and there is an explicit isospin dependence. To perform a study in asymmetric nuclear matter, the nonlinearity of the energy gap on the isospin degree of freedom should be considered [22] and may be studied in a forthcoming study.

The pairing gap in uniform matter is obtained from the BCS gap equation [23]:

$$
\Delta_{k}=\sum_{k^{\prime}}-v_{k k^{\prime}} \frac{\Delta_{k^{\prime}}}{2 E_{k^{\prime}}},
$$

solved under the condition of the particle number conservation. In a given volume $V$, one assumes constant density, given by

$$
\rho_{\tau}=\frac{2}{V} \sum_{k}\left[1-\frac{e_{\tau}(k)-\mu_{\tau}}{E_{k, \tau}}\right],
$$



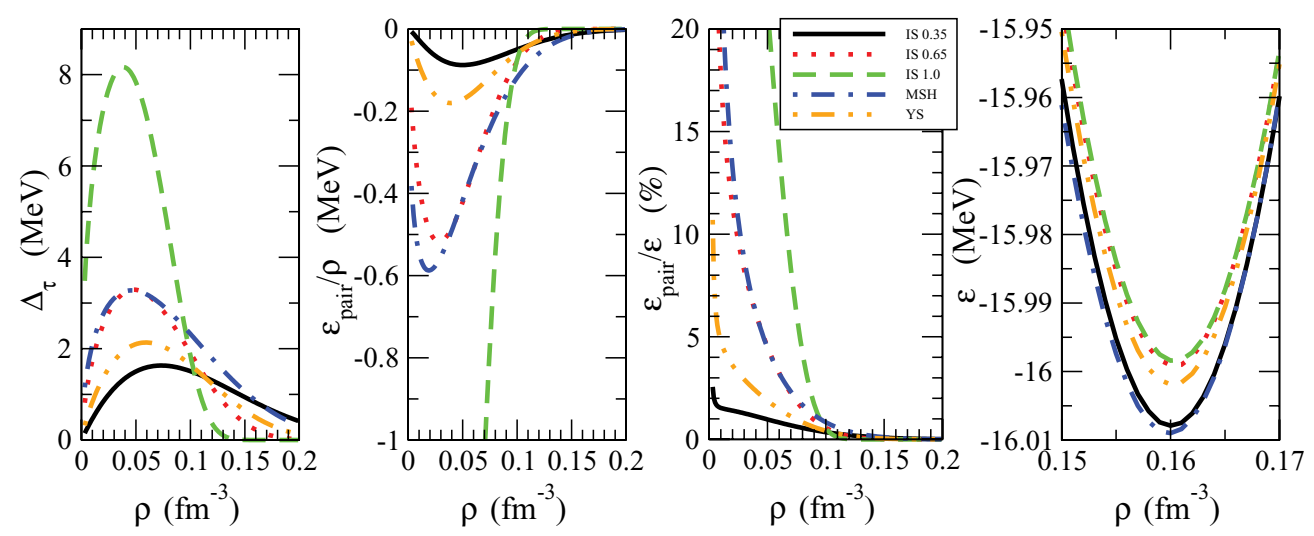

FIG. 1. (Color online) Pairing gap $\Delta_{\tau}$, pairing energy per particle $\epsilon_{\text {pair }} / \rho$, percentage of pairing energy with respect to the total energy $\epsilon$, and equation of state around the saturation point in symmetric matter obtained with various pairing interactions employed in connection with the SLy5 Skyrme interaction. The solid, dotted, and dashed lines correspond to the IS pairing interactions with $\eta=0.35,0.65$, and 1.0 , respectively, in Eq. (3). The dash-dotted and dash-dotted-dotted lines show the results of IS + IV interactions in Ref. [15] (MSH) and Ref. [16] (YS), respectively. See the text for details.

where the quasiparticle energy is defined as $E_{k, \tau}=$ $\sqrt{\left(e_{\tau}(k)-\mu_{\tau}\right)^{2}+\Delta_{k, \tau}^{2}}, e_{\tau}(k)$ is the single particle energy, and $\mu_{\tau}$ is the chemical potential. In Eq. (6), $v_{k k^{\prime}}$ is the pairing matrix element for the plane waves, namely, $\left\langle k \bar{k}|v| k^{\prime} \bar{k}^{\prime}\right\rangle$. Notice that in the case of the zero-range pairing interaction, the pairing gap $\Delta_{k}$ is independent of $k$.

In Fig. 1, we display the pairing gap $\Delta_{\tau}$, the pairing energy per particle $\epsilon_{\text {pair }} / \rho$, and the percentage of the pairing energy with respect to the total energy $\epsilon$ in symmetric matter for the various pairing interactions together with the SLy5 Skyrme interaction in the mean field channel. There is a critical density $\rho_{c} \approx 0.11 \mathrm{fm}^{-3}$ at which all the pairing interactions give almost the same result for the pairing gap. This has already been noticed in Ref. [13] and may be related to the fact that in fitting the two-neutron separation energy, one is sensitive to the space region of the nuclear surface, where the density is somewhat lower than the saturation density; therefore the pairing gap is constrained at $\rho_{c}$ rather than at $\rho_{0}$. Notice that the parameters of the MSH pairing interaction have been determined without using constraints from finite systems, and $\Delta$ at $\rho_{0}$ does not necessarily coincide with the one from the other pairing interactions. Above $\rho_{c}$, the more surface type the pairing interaction (i.e., the larger the $\eta$ that is taken), the smaller the pairing gap $\Delta_{\tau}$. Below the critical density, the trend is reversed: The more surface type the pairing interaction is, the larger the pairing gap. The contribution of the pairing energy is increased at low densities. Around the saturation density, the pairing energy per particle is much smaller than the binding energy $(-16 \mathrm{MeV})$.

\section{B. Incompressibility and symmetry energy}

The compressibility $\chi$ is usually defined by

$$
\chi=-\frac{1}{V} \frac{\partial V}{\partial P}=\frac{1}{\rho}\left(\frac{\partial P}{\partial \rho}\right)^{-1},
$$

and the pressure is related to the energy density $\epsilon$ by

$$
P=-\frac{\partial E}{\partial V}=\rho^{2} \frac{\partial E / A}{\partial \rho}=\rho \frac{\partial \epsilon}{\partial \rho}-\epsilon=\rho \mu-\epsilon .
$$

The chemical potential is defined by

$$
\mu=\frac{\partial E}{\partial A}=\frac{\partial \epsilon}{\partial \rho} .
$$

From Eqs. (8) and (9), we obtain

$$
\frac{1}{\chi}=\rho^{2} \frac{\partial^{2} \epsilon}{\partial \rho^{2}}
$$

The compressibility $\chi(\rho)$ is a function of the density $\rho$ and the asymmetry parameter $\delta$. Furthermore, one defines the incompressibility at the saturation density in symmetric nuclear matter by

$$
K_{\infty}=\left.k_{F}^{2} \frac{\partial^{2} E / A}{\partial k_{F}^{2}}\right|_{k_{F \infty}}=\left.9 \rho_{0}^{2} \frac{\partial^{2} E / A}{\partial \rho^{2}}\right|_{\rho_{0}} .
$$

The relation between $K_{\infty}$ and $\chi$ is given by

$$
K_{\infty}=\frac{9}{\rho_{0} \chi\left(\rho_{0}\right)} .
$$

It is worth keeping in mind that the incompressibility $K_{\infty}$ is defined only at the saturation density $\rho_{0}$, and in particular, Eq. (13) is valid only for $\rho=\rho_{0}$. Indeed, more generally, we can define the density-dependent incompressibility (or bulk modulus) as [24]

$$
K(\rho)=\frac{9}{\rho \chi}=9 \rho^{2} \frac{\partial^{2} E / A}{\partial \rho^{2}}+\frac{18}{\rho} P,
$$

which coincides with the incompressibility $K_{\infty}$ at the saturation density.

The symmetry energy $S(\rho)$ is defined by

$$
S(\rho)=\left.\frac{1}{2} \frac{\partial^{2} \epsilon / \rho}{\partial \delta^{2}}\right|_{\delta=0} .
$$

The symmetry energy can be expanded, around the saturation density, as

$$
S(\rho)=J+L\left(\frac{\rho-\rho_{0}}{3 \rho_{0}}\right)+\frac{1}{2} K_{\mathrm{sym}}\left(\frac{\rho-\rho_{0}}{3 \rho_{0}}\right)^{2}
$$



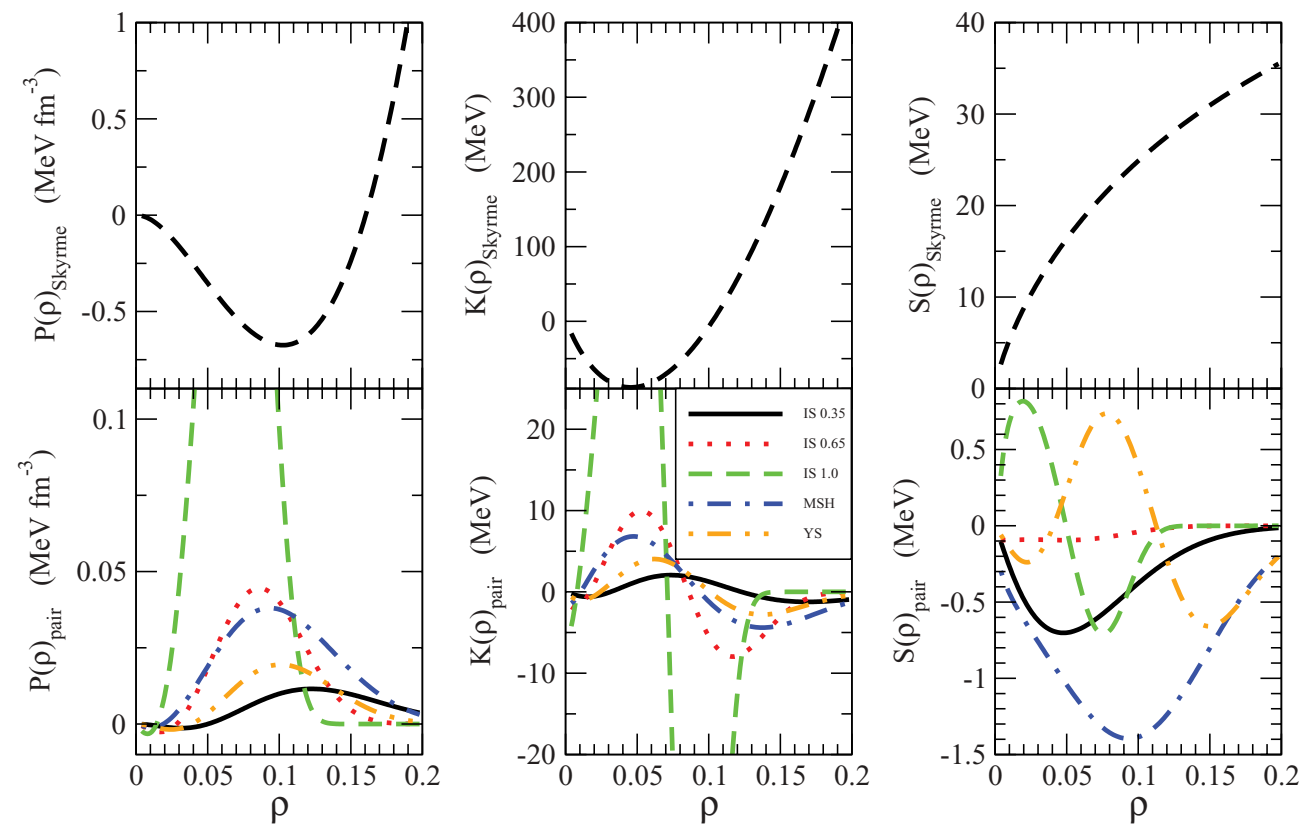

FIG. 2. (Color online) (top) Pressure, incompressibility, and symmetry energy without pairing, and (bottom) the contributions of various pairing interactions to these quantities. The HF energy $\epsilon_{\text {Skyrme }}$ is calculated using the SLy5 interaction. For details, see the caption to Fig. 1 and the text.

where $J$ is defined by $J=S\left(\rho_{0}\right), L=\left.\left(3 \rho_{0}\right)(\partial S / \partial \rho)\right|_{\rho_{0}}$, and $K_{\text {sym }}=\left.\left(9 \rho_{0}^{2}\right)\left(\partial^{2} S / \partial \rho^{2}\right)\right|_{\rho_{0}}$.

\section{Results}

Figure 2 displays the pressure $P$ in Eq. (9), the incompressibility $K(\rho)$ in Eq. (14), and the symmetry energy $S(\rho)$ in Eq. (16) without pairing (top), and the contribution of pairing to these quantities (bottom), using the SLy5 interaction. This contribution is calculated with the same equations, but considering only the pairing term of the energy density in Fig. 1. The same pairing interactions have been considered here as in Fig. 1. Close to the saturation density, the contribution from pairing is very small. This is also illustrated in Table II: The pairing interaction has small effects at the saturation density. In the case of the incompressibility $K_{\infty}$, pairing can still produce a few percentage effect (e.g., $K_{\infty}$ is changed from $230.2 \mathrm{MeV}$ to $223.9 \mathrm{MeV}$ in the case of the MSH pairing interaction). The MSH, YS, IS 0.35 pairing interactions

TABLE II. Properties of nuclear matter for various pairing interactions. The SLy5 Skyrme force is used for the mean field.

\begin{tabular}{lcccccr}
\hline \hline Pairing & $\begin{array}{c}\rho_{0} \\
\left(\mathrm{fm}^{-3}\right)\end{array}$ & $\begin{array}{c}\mathrm{E} / \mathrm{A}\left(\rho_{0}\right) \\
(\mathrm{MeV})\end{array}$ & $\begin{array}{c}K_{\infty} \\
(\mathrm{MeV})\end{array}$ & $\begin{array}{c}J \\
(\mathrm{MeV})\end{array}$ & $\begin{array}{c}L \\
(\mathrm{MeV})\end{array}$ & $\begin{array}{c}K_{\text {sym }} \\
(\mathrm{MeV})\end{array}$ \\
\hline No pairing & 0.1604 & -15.999 & 230.2 & 32.03 & 48.25 & -112.3 \\
IS $\eta=0.35$ & 0.1601 & -15.998 & 227.3 & 31.93 & 48.49 & -129.7 \\
IS $\eta=0.65$ & 0.1603 & -15.998 & 228.1 & 32.02 & 48.30 & -113.7 \\
IS $\eta=1.00$ & 0.1604 & -15.999 & 230.1 & 32.03 & 48.25 & -112.3 \\
MSH & 0.1599 & -15.998 & 223.9 & 31.33 & 55.77 & -139.7 \\
YS & 0.1602 & -15.998 & 227.0 & 31.39 & 52.04 & 13.2 \\
\hline \hline
\end{tabular}

modify the incompressibility by 3 to $6 \mathrm{MeV}$, that is, by about $2 \%$. It should be noted that at the saturation density, the contribution to the slope parameters of the symmetry energy, $L$, and $K_{\text {sym }}$ of the interactions MSH and YS is larger than that of the other IS forces. The effects on $L$ can be about $15 \%$, whereas $K_{\text {sym }}$ can be modified in an important way. This is related to the dependence of these pairing interactions on the isovector density.

However, at lower densities, the pairing effects become appreciably larger, as seen in Fig. 2. In the case of the pure surface pairing, there are important contributions to the pressure, incompressibility, and symmetry energy: These quantities can be strongly affected by pairing, which can lead to variations up to about a factor of 2 . Other pairing interactions also provide significant corrections to the pressure and the incompressibility, typically, around $10 \%$. In the case of the symmetry energy, below $\rho \approx 0.1 \mathrm{fm}^{-3}$, the IS + IV pairing interactions (MSH and YS) predict an opposite and positive contribution compared to the negative contributions of IS pairing interactions. It should be noted that the pairing contribution to these quantities is generally larger at densities below saturation.

To obtain a more general view of the pairing effect on the incompressibility, Table III displays the $K_{\infty}$ values obtained for SLy5, LNS, Sk255, and Sk272 Skyrme functionals, with various pairing interactions. The correction induced by the pairing interaction IS 0.35 is the largest among the IS interactions and reduces the incompressibility $K_{\infty}$ by about $3 \mathrm{MeV}$. The MSH interaction induces a correction of $6.3 \mathrm{MeV}$ on the incompressibility (Table II). It should be noted that the pure surface pairing interaction provides no modification of $K_{\infty}$. Depending on the Skyrme models, there shall also be an effect because of the different effective masses $m^{*} / m$, 
TABLE III. Nuclear matter incompressibility $K_{\infty}(\mathrm{MeV})$ for SLy5 [12], LNS [15], Sk255 [14], and Sk272 [14] Skyrme functionals. The dependence of $K_{\infty}$ on the pairing interaction is displayed: mixed (IS $\eta=0.35$ ) and surface (IS $\eta=1.00$ ).

\begin{tabular}{lcccc}
\hline \hline Pairing & SLy5 & LNS & Sk255 & Sk272 \\
\hline No pairing & 230.2 & 211.0 & 255.2 & 271.8 \\
IS $\eta=0.35$ & 227.3 & 208.4 & 251.3 & 268.3 \\
IS $\eta=1.00$ & 230.1 & 211.0 & 255.2 & 271.8 \\
\hline \hline
\end{tabular}

but they are incorporated in the renormalization of the pairing interaction parameter $v_{0}$ shown in Table I.

It is expected that the preceding pairing effects at low densities may also affect finite nuclei. In the case of incompressibility, we can define a finite nucleus value $K_{A}$ and expect that this value will be affected by the pairing more than $K_{\infty}$ because of the presence of a lower density region, that is, the nuclear surface. We analyze this point in the next section, and we argue that a similar reasoning holds for the symmetry energy.

\section{LOCAL DENSITY APPROXIMATION}

This section relates the general expressions in uniform matter obtained in Sec. II with the observables in finite nuclei in the LDA. The aim is to estimate the role of pairing in the incompressibility and symmetry energy of finite nuclei in a simple and transparent way. The validity of the LDA will be estimated by comparing the predicted nuclei incompressibility with the one obtained by a microscopic approach.

The binding energy per nucleon in the LDA reads

$$
B_{A}(N, Z)=B_{\text {Nucl }}(N, Z)+B_{\text {Coul }} \frac{Z^{2}}{A^{4 / 3}},
$$

where $B_{\text {Nucl }}(N, Z)$ includes the bulk, surface, and pairing contributions. It is defined by

$$
B_{\mathrm{Nucl}}(N, Z)=\frac{1}{A} \int d^{3} r \in(r),
$$

where $\epsilon(r)=\epsilon\left[\rho_{n}(r), \rho_{p}(r)\right]=\epsilon_{\text {Skyrme }}(r)+\epsilon_{\text {pair }}(r)$, as was defined in Eq. (1). The neutron and proton densities $\rho_{n}(r), \rho_{p}(r)$ can be obtained, in the present context, by means of a spherical HF calculation. The pairing contribution to the binding energy is defined by

$$
B_{\text {pair }}(N, Z)=\frac{1}{A} \int d^{3} r \epsilon_{\text {pair }}(r) .
$$

$B_{\text {Nucl }}$ can be expanded around the saturation density,

$$
B_{\mathrm{Nucl}}(N, Z) \approx B_{\infty}+\frac{1}{2} K_{A}\left(\frac{\rho-\rho_{0}}{3 \rho_{0}}\right)^{2}+S_{A} \delta^{2},
$$

where the incompressibility in nuclei, $K_{A}$, is defined by $K_{A}=$ $K_{\text {Nucl }}+K_{\text {Coul }} \cdot Z^{2} A^{-4 / 3}$, and

$$
K_{\text {Nucl }}=9 \rho_{0}^{2} \frac{\partial^{2} B_{\mathrm{Nucl}}(N, Z)}{\partial \rho^{2}},
$$

while the pairing contribution to the incompressibility is defined by

$$
K_{\text {pair }}=9 \rho_{0}^{2} \frac{\partial^{2} B_{\text {pair }}(N, Z)}{\partial \rho^{2}} .
$$

The Coulomb contribution, $K_{\text {Coul }}$, can be evaluated using, for instance, the Thomas-Fermi approximation [cf. Eq. (A1) in Ref. [25]]. It will not be included in the present work, but the value obtained in Ref. [25] is $-8 \mathrm{MeV}<K_{\text {Coul }}<-4 \mathrm{MeV}$, depending on the interaction that is used.

The symmetry energy in finite nuclei, $S_{A}$, is defined by

$$
S_{A}=\left.\frac{1}{2} \frac{\partial^{2} B_{\mathrm{Nucl}}(N, Z)}{\partial \delta^{2}}\right|_{\delta=0},
$$

and the contribution of the pairing correlations to $S_{A}$ is defined by

$$
S_{\text {pair }}=\left.\frac{1}{2} \frac{\partial^{2} B_{\text {pair }}(N, Z)}{\partial \delta^{2}}\right|_{\delta=0} .
$$

Introducing the mass formula (18) into Eq. (21), one obtains

$$
K_{\mathrm{Nucl}}=\frac{\rho_{0}}{A} \int d^{3} r K_{\mathrm{Nucl}}(r),
$$

with

$$
K_{\mathrm{Nucl}}(r)=\frac{\rho_{0}}{\rho} K[\rho(r)] .
$$

For small values of the density $\left(\rho \lesssim 0.6 \rho_{0}\right.$, i.e., $\mathrm{r} \gtrsim 5 \mathrm{fm}$ in ${ }^{120} \mathrm{Sn}$ ), the incompressibility is found to be negative: This is because of the spinodal instability in nuclear matter that is not present in finite systems [26]. For this reason, the integral (25) is limited to the region where $K_{\text {Nucl }}(r)$ is positive. In this way, the spurious component because of the spinodal instability is removed.

Introducing the quantity

$$
S_{A}(r)=\left.\frac{1}{2 \rho_{0}} \frac{\partial^{2} \epsilon}{\partial \delta^{2}}\right|_{\delta=0}=\frac{\rho}{\rho_{0}} S[\rho(r)],
$$

the symmetry energy in nuclei (23) reads

$$
S_{A}=\frac{\rho_{0}}{A} \int d^{3} r S_{A}(r) .
$$

We first perform a self-consistent HF calculation that provides the neutron and proton densities in ${ }^{120} \mathrm{Sn}$. From these densities, we deduce the radial distributions of the mean field part and pairing part of $\epsilon(r)$ given in Eq. (1), $K_{\text {Nucl }}(r)$ given in Eq. (26), and $S_{A}(r)$ given in Eq. (27); these radial functions are shown in Fig. 3. As expected from the results discussed in the previous section, the pairing effects on $\epsilon(r), K_{\mathrm{Nucl}}(r)$, and $S_{A}(r)$ come from the low-density surface region.

From Eqs. (18), (25), and (28), we obtain, in the SLy5 case, $B_{A}=-13.5 \mathrm{MeV}, K_{\mathrm{Nucl}}=119.8 \mathrm{MeV}$, and $S_{A}=25.7 \mathrm{MeV}$ without the contribution from the pairing correlations. The Coulomb contribution has not been included. The value for $K_{\text {Nucl }}$ should be compared with that of $141 \mathrm{MeV}$ obtained by the constrained HFB (CHFB) calculations presented in the next section (cf. Fig. 6). It should be noted that the CHFB calculations take into account the contribution coming from 

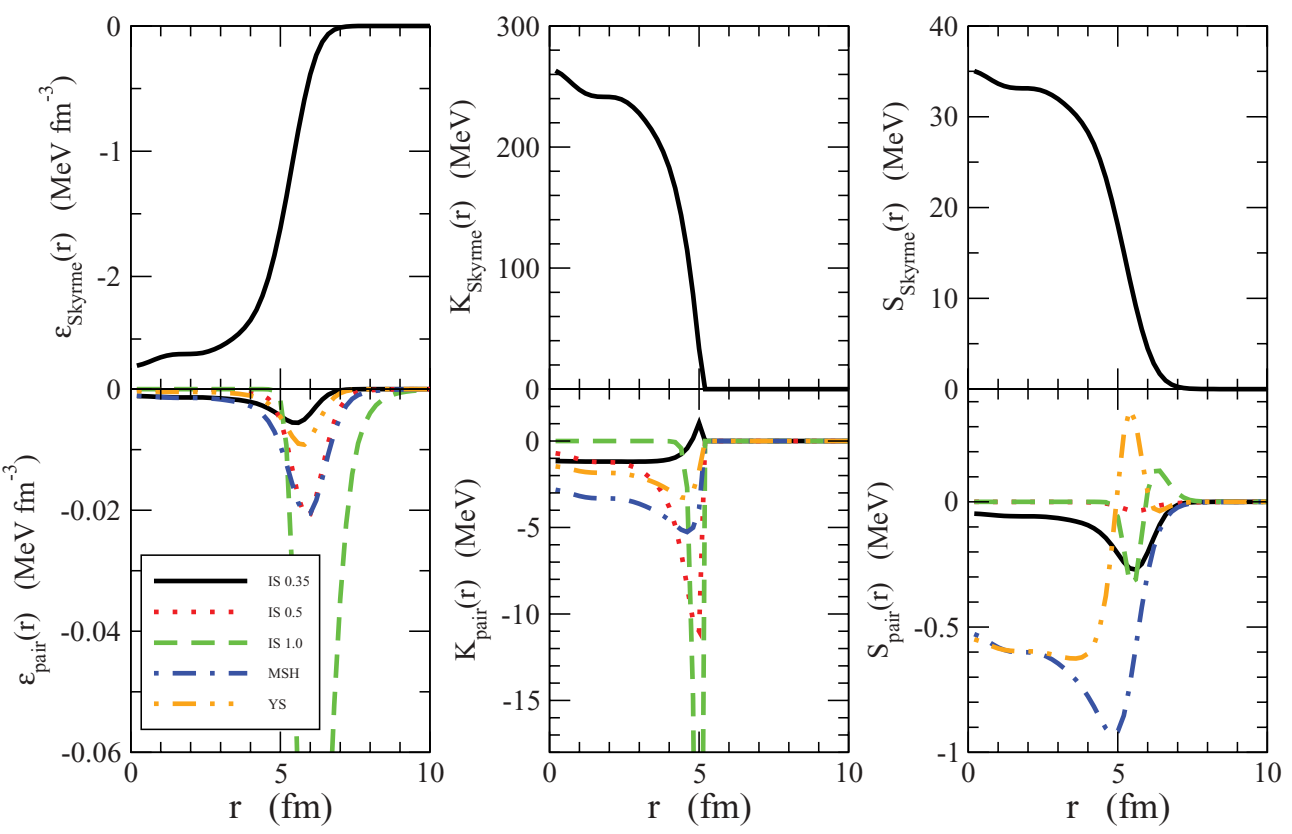

FIG. 3. (Color online) (bottom) Radial dependence of $\epsilon(r), K_{\mathrm{Nucl}}(r)$, and $S_{A}(r)$ for the various pairing interactions considered using the SLy5 force in ${ }^{120} \mathrm{Sn}$ within the LDA. (top) The contribution of the Skyrme term plus the kinetic term. See the caption to Fig. 1 and the text for details.

the Coulomb interaction. This contribution is estimated to be about $20 \mathrm{MeV}$ in ${ }^{120} \mathrm{Sn}$, using the values of $K_{\mathrm{Coul}}$ from Ref. [25]. The good agreement between the LDA and the CHFB results ensures that LDA provides a sound framework to relate the nuclear matter incompressibility and the finite nucleus one.

The contributions of pairing correlations to the binding energy, the bulk modulus, and the symmetry energy are shown in Table IV for the various pairing interactions considered. The contribution of the surface-type pairing (IS $\eta=1.0$ ) reduces $K_{A}$ by about $5 \%$, whereas, for the IS mixed-type pairing $(\eta=0.35$ or 0.65$)$ and the IS + IV (MSH and YS) pairing interactions, the effect on $K_{A}$ is predicted to be smaller. In Table IV, it is also observed that pairing effects affect the binding energy by few percent, up to $5 \%$ for the surface-type pairing interaction. For the symmetry energy, pairing effects are negligible, being below $1 \%$, except for the IS + IV pairing (MSH).

TABLE IV. Contributions of pairing correlations to the binding energy, the incompressibility, and the symmetry energy in ${ }^{120} \mathrm{Sn}$. The mean field is calculated by using the SLy5 interaction.

\begin{tabular}{lccc}
\hline \hline Pairing & $\begin{array}{c}B_{\text {pair }} \\
(\mathrm{MeV})\end{array}$ & $\begin{array}{c}K_{\text {pair }} \\
(\mathrm{MeV})\end{array}$ & $\begin{array}{c}S_{\text {pair }} \\
(\mathrm{MeV})\end{array}$ \\
\hline IS $\eta=0.35$ & -0.03 & -0.5 & -0.25 \\
IS $\eta=0.65$ & -0.11 & -3.9 & -0.03 \\
IS $\eta=1.00$ & -0.64 & -6.0 & -0.03 \\
MSH & -0.13 & -3.2 & -0.93 \\
YS & -0.05 & -1.9 & -0.24 \\
\hline \hline
\end{tabular}

\section{FINITE NUCLEI}

In the previous section, the LDA has shown that the pairing effect on the symmetry energy is negligible, whereas appreciable effects are observed in the case of the incompressibility. In this section, the role of pairing effects on the finite nucleus incompressibility $K_{A}$ is investigated using a microscopic approach.

We use the sum rule approach to calculate the centroid energy of the isoscalar GMR. It is known that the finite nucleus incompressibility $K_{A}$ is related to that centroid energy by means of the relation

$$
E_{\mathrm{ISGMR}}=\sqrt{\frac{\hbar^{2} K_{A}}{m\left\langle r^{2}\right\rangle}},
$$

where $m$ is the nucleon mass and $\left\langle r^{2}\right\rangle$ denotes the groundstate expectation value of the square radius. In a microscopic approach, for the so-called scaling $K_{A}$, we calculate the energy as

$$
E_{\mathrm{ISGMR}}=\sqrt{\frac{m_{1}}{m_{-1}}},
$$

where the $k$ th energy weighted sum rule is defined as

$$
m_{k}=\sum_{i} E_{i}^{k}|\langle i|\hat{Q}| 0\rangle|^{2},
$$

with the RPA excitation energy $E_{i}$ and the isoscalar monopole transition operator

$$
\hat{Q}=\sum_{i=1}^{A} r_{i}^{2}
$$


The $m_{1}$ moment is evaluated by the double commutator using the Thouless theorem [27]:

$$
m_{1}=\frac{2 \hbar^{2}}{A}\left\langle r^{2}\right\rangle .
$$

In the present HFB calculations, the energy cutoff is $60 \mathrm{MeV}$, and the $j_{\max }$ value is $15 / 2$ in the case of IS pairing and is extended to $25 / 2$ for the IS + IV MSH pairing, which are the cutoffs used in the design of these pairing interactions $[13,16]$, to ensure convergence of the results. It should be noted that the strength $v_{0}$ of the IS pairing interactions is adjusted in nuclei for its corresponding $j_{\max }$ and energy cutoff. Therefore the different values of $j_{\max }$ between IS and the IS + IV pairing have little influence on the calculations.

Concerning the evaluation of the $m_{-1}$ moment, the CHFB approach is used. It should be noted that the extension of the constrained HF method [2,28] to the CHFB case has been recently demonstrated in Ref. [29] and employed also in Ref. [6]. The CHFB Hamiltonian is built by adding the constraint associated with the IS monopole operator, namely,

$$
\hat{H}_{\text {constr }}=\hat{H}+\lambda \hat{Q} \text {, }
$$

and the $m_{-1}$ moment is obtained from the derivative of the expectation value of the monopole operator on the CHFB solution $|\lambda\rangle$ :

$$
m_{-1}=-\frac{1}{2}\left[\frac{\partial}{\partial \lambda}\langle\lambda|\hat{Q}| \lambda\rangle\right]_{\lambda=0} .
$$

We first investigate the correlations between nuclear matter incompressibility $K_{\infty}$ and finite nucleus $K_{A}$, in the absence of pairing correlations. This correlation has been found in all the previous articles on the subject but is reported here as a benchmark for further considerations concerning the effect of pairing. For this purpose, we choose the doubly magic ${ }^{208} \mathrm{~Pb}$ nucleus. Figure 4 displays $K_{A}$ (obtained with the CHF method) versus $K_{\infty}$ for the four Skyrme interactions LNS, SLy5, Sk255, and Sk272. These four interactions span a large range of incompressibilities and have been fitted using different physics inputs: the neutron matter EOS from realistic forces in the case of SLy5, Brückner-HF calculations in nuclear matter in

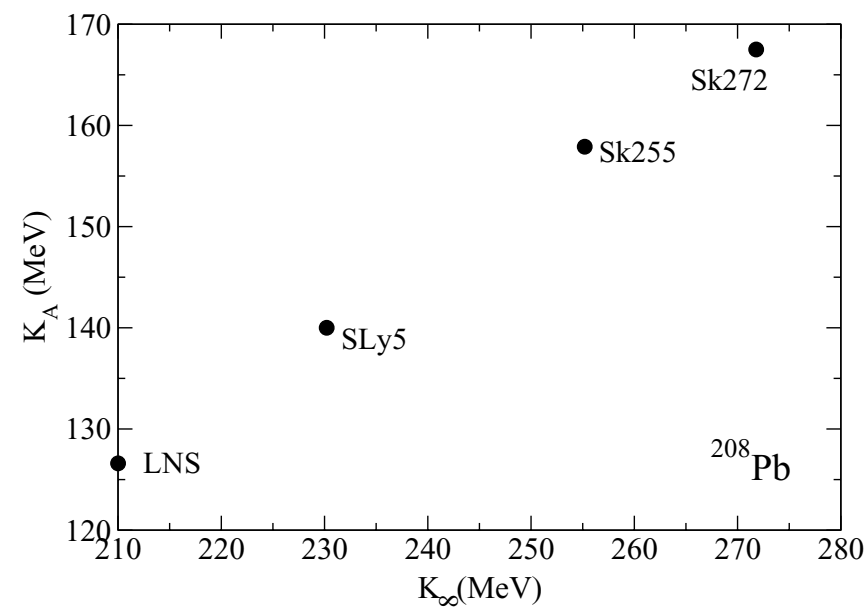

FIG. 4. $K_{\infty}$ vs $K_{A}$ for ${ }^{208} \mathrm{~Pb}$ obtained by the CHF method for several Skyrme interactions.

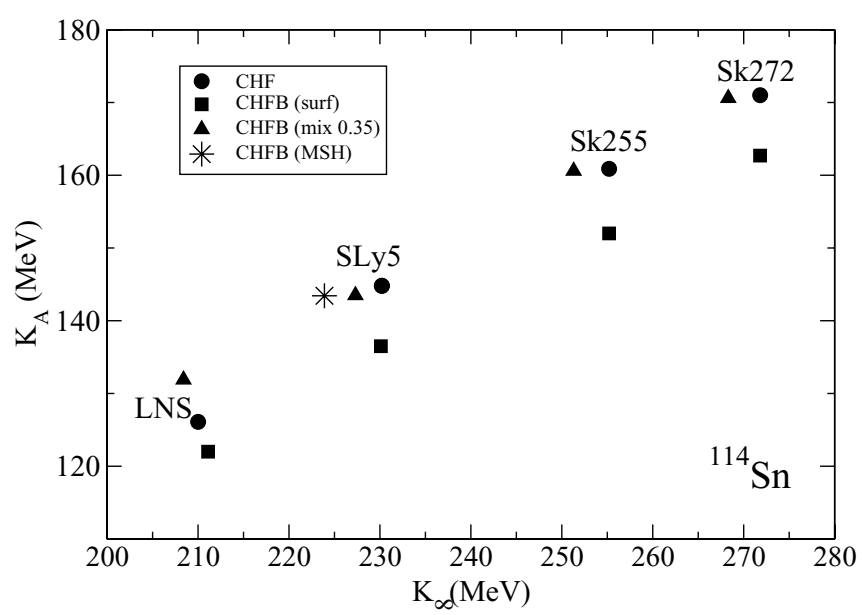

FIG. 5. $K_{\infty}$ vs $K_{A}$ for ${ }^{114} \mathrm{Sn}$ obtained by the CHF and the CHFB method with surface-type and mixed-type pairing interactions for several Skyrme interactions.

the case of LNS, and the empirical properties of symmetric uniform matter plus a few binding energies and charge radii of selected nuclei (the same that had been used to fit some relativistic functionals like NL3) in the case of Sk255 and Sk272. In this sense, these interactions provide representative samples of the Skyrme functionals.

It should be stressed that both $K_{A}$ and $K_{\infty}$ are here evaluated consistently in a microscopic model. As expected, $K_{A}$ is clearly correlated with $K_{\infty}$. To find the pairing effects, we also study the case of the open-shell nuclei ${ }^{114} \mathrm{Sn}$ and ${ }^{120} \mathrm{Sn}$ in Figs. 5 and 6, respectively. For each Skyrme interaction, three results are shown: (1) the CHF result without pairing, (2) the CHFB result using the surface-type pairing interaction, and (3) the CHFB result using the mixed-type $(\eta=0.35)$ pairing interaction. In the case of SLy5, the IS + IV MSH pairing interaction is also used. The surface-type interaction decreases the finite nucleus incompressibility $K_{A}$ by about $10 \%$ in ${ }^{114} \mathrm{Sn}$ and $5 \%$ in ${ }^{120} \mathrm{Sn}$, whereas the mixed-type pairing interaction has a negligible effect on $K_{A}$. Conversely, it should be noted that the mixed pairing interaction has some effect on $K_{\infty}$, whereas the pure surface pairing interaction has a

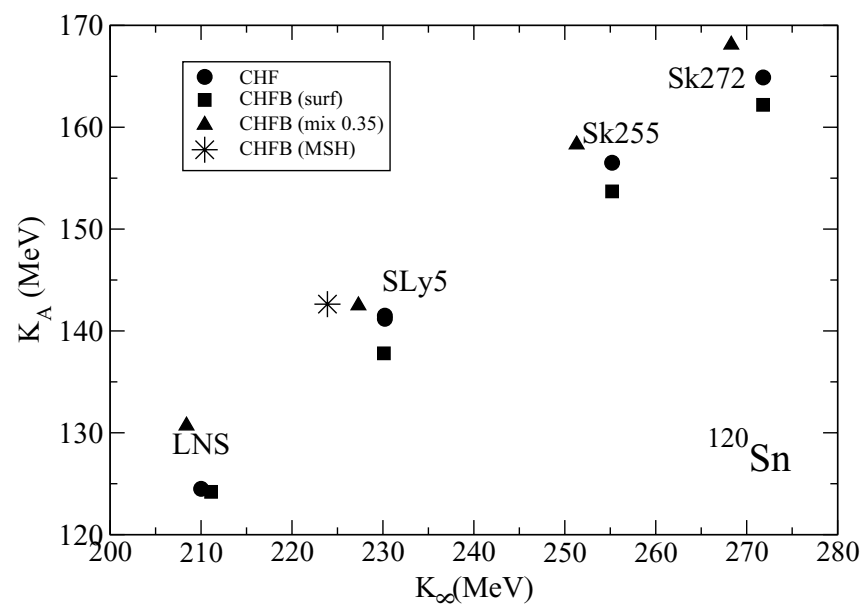

FIG. 6. Same as Fig. 5, but for ${ }^{120} \mathrm{Sn}$. 
negligible effect on $K_{\infty}$, as seen in Fig. 5. In the case of LNS, the reduction of $K_{A}$ is smaller for the surface pairing, and an increase of $K_{A}$ is even observed for the mixed pairing case. In the SLy5 case, predictions using the IS + IV MSH pairing interactions show no variation of $K_{A}$ but affect $K_{\infty}$.

To study how these conclusions in ${ }^{114} \mathrm{Sn}$ are sensitive to the nuclear shell structure, the results for ${ }^{120} \mathrm{Sn}$ are displayed in Fig. 6, where pairing effects are expected to be smaller than in ${ }^{114} \mathrm{Sn}$ because of the subshell closure. In this case, the reduction of $K_{A}$ because of the surface-type pairing effect drops to $5 \%$. For the mixed pairing interaction, a small increase of $K_{A}$ is observed. This feature is again more pronounced in the LNS case. In the SLy5 case, predictions using the IS + IV MSH pairing interactions show no variation of $K_{A}$ but affect $K_{\infty}$. It should be noted that similar trends are observed with the LDA predictions (see Table IV). Also, they are consistent with previous studies $[5,6]$. To further study shell effects, the same calculations have been performed on ${ }^{126} \mathrm{Sn}$, and a similar pattern to Fig. 6 is found, showing that the present results are rather independent from shell effects in open-shell nuclei.

Evidently, the pairing effects tend to decrease the finite nucleus incompressibility $K_{A}$ in the surface pairing case, whereas $K_{\infty}$ is decreased in the mixed pairing case. Hence the question of constraining the pairing interaction through precise (typical resolution of few hundreds of $\mathrm{keV}$ ) GMR measurement is raised. The systematic softness of open-shell $\mathrm{Sn}$ and $\mathrm{Cd}$ isotopes measured recently [30-32] through the energy of the GMR might be the sign of a surface pairing interaction at work [6].

\section{CONCLUSIONS}

The effect of superfluidity on the incompressibility has been studied in both nuclear matter and finite nuclei using various pairing functionals. A small effect is observed on the nuclear matter incompressibility at the saturation density and the symmetry energy, but it is non-negligible on $L$ and $K_{\text {sym }}$.

However, at lower density, the pairing effect on the incompressibility is significant and can have an substantial impact on neutron star studies or on the interpretation of multifragmentation data. It has been shown that the LDA provides a relevant framework for a qualitative understanding and interpretation of the microscopic results. The effect of the pairing correlations is localized near the surface of nuclei, and the effect of the pairing correlations is to make slightly softer nuclear EOS. Especially in the low-density region in nuclear matter, the pairing effect is more noticeable. This may explain why such effects are expected to happen in the surface of the finite nuclei. In the case of the IS + IV pairing interaction, no strong effect is observed on $K_{A}$. In general, the pairing effects on the finite nucleus incompressibility $K_{A}$ are more important when the interaction is more surface type (larger $\eta$ value).

This study shows that with respect to current experimental uncertainties, the pairing effects should be considered when extracting the incompressibility value from GMR data, which can now reach an accuracy of several hundreds of keV [30]. Experimentally, it would be useful to measure the GMR on isotopic chains, including both open-shell and doubly magic nuclei such as ${ }^{132} \mathrm{Sn}$. Such measurements are starting to be undertaken [30-32] and will be extended to unstable nuclei [33].

\section{ACKNOWLEDGMENTS}

This work was supported by the Japanese Ministry of Education, Culture, Sports, Science, and Technology by a Grant-in-Aid for Scientific Research under Program No. 20540277 and No. 22540262, by an ANR NExEN contract, and by CompStar, a Research Networking Programme of the European Science Foundation.
[1] J.-P. Blaizot, Phys. Rep. 64, 171 (1980).

[2] G. Colò, N. V. Giai, J. Meyer, K. Bennaceur, and P. Bonche, Phys. Rev. C 70, 024307 (2004).

[3] L. Trippa, G. Colò, and E. Vigezzi, Phys. Rev. C 77, 061304(R) (2008).

[4] O. Civitarese, A. G. Dumrauf, M. Reboiro, P. Ring, and M. M. Sharma, Phys. Rev. C 43, 2622 (1991).

[5] J. Li, G. Colò, and J. Meng, Phys. Rev. C 78, 064304 (2008).

[6] E. Khan, Phys. Rev. C 80, 011307(R) (2009).

[7] E. Khan, Phys. Rev. C 80, 057302 (2009).

[8] G. F. Bertsch, C. A. Bertulani, W. Nazarewicz, N. Schunck, and M. V. Stoitsov, Phys. Rev. C 79, 034306 (2009).

[9] A. W. Steiner, M. Prakash, J. M. Lattimer, and P. J. Ellis, Phys. Rep. 411, 325 (2005).

[10] B. A. Li, L.-W. Chen, and C. M. Ko, Phys. Rep. 464, 113 (2008).

[11] A. Klimkiewicz et al., Phys. Rev. C 76, 051603(R) (2007); A. Carbone, G. Colò, A. Bracco, L. G. Cao, P. F. Bortignon, F. Camera, and O. Wieland, ibid. 81, 041301 (2010).

[12] E. Chabanat, P. Bonche, P. Haensel, J. Meyer, and R. Schaeffer, Nucl. Phys. A 635, 231 (1998).

[13] E. Khan, M. Grasso, and J. Margueron, Phys. Rev. C 80, 044328 (2009).
[14] B. K. Agrawal, S. Shlomo, and V. K. Au, Phys. Rev. C 68, 031304(R) (2003).

[15] L. G. Cao, U. Lombardo, C. W. Shen, and N. V. Giai, Phys. Rev. C 73, 014313 (2006).

[16] J. Margueron, H. Sagawa, and K. Hagino, Phys. Rev. C 76, 064316 (2007).

[17] M. Yamagami, Y. R. Shimizu, and T. Nakatsukasa, Phys. Rev. C 80, 064301 (2009).

[18] L. N. Cooper, R. L. Mills, and A. M. Sessler, Phys. Rev. 114, 1377 (1959).

[19] E. Garrido, P. Sarriguren, E. Moya de Guerra, and P. Schuck, Phys. Rev. C 60, 064312 (1999).

[20] M. Baldo, M. Farine, U. Lombardo, E. E. Saperstein, P. Schuck, and M. V. Zverev, Eur. Phys. J. A 18, 17 (2003).

[21] S. S. Pankratov, M. Baldo, U. Lombardo, E. E. Saperstein, and M. V. Zverev, Nucl. Phys. A 765, 61 (2006).

[22] S. S. Zhang, L. G. Cao, U. Lombardo, E. G. Zhao, and S. G. Zhou, Phys. Rev. C 81, 044313 (2010).

[23] J. Bardeen, L. N. Cooper, and J. R. Schrieffer, Phys. Rev. 108, 1175 (1957).

[24] A. L. Fetter and J. D. Walecka, Quantum Theory of ManyParticle Systems (McGraw-Hill, New York, 1971). 
[25] H. Sagawa, S. Yoshida, G.-M. Zeng, J.-Z. Gu, and X.-Z. Zhang, Phys. Rev. C 76, 034327 (2007).

[26] J. Margueron and P. Chomaz, Phys. Rev. C 67, 041602(R) (2003).

[27] D. J. Thouless, Nucl. Phys. 22, 78 (1961).

[28] O. Bohigas, A. M. Lane, and J. Martorell, Phys. Rep. 51, 267 (1979).
[29] L. Capelli, G. Colò, and J. Li, Phys. Rev. C 79, 054329 (2009).

[30] T. Li et al., Phys. Rev. Lett. 99, 162503 (2007).

[31] T. Li et al., Phys. Rev. C 81, 034309 (2010).

[32] U. Garg, in COMEX3 Conference (MSU, Mackinac Island, 2009).

[33] C. Monrozeau et al., Phys. Rev. Lett. 100, 042501 (2008). 